\title{
Reciprocal negative feedback loop between EZH2 and miR-101-1 contributes to miR-101 deregulation in hepatocellular carcinoma
}

\author{
DA HUANG ${ }^{*}$, XIAOBEI WANG ${ }^{*}$, CHUNBO ZHUANG, WUHE SHI, MU LIU, \\ QIMING TU, DETAI ZHANG and LIHUA HU

\begin{abstract}
Department of Clinical Laboratory, Wuhan Union Hospital, Tongji Medical College, Huazhong University of Science and Technology, Wuhan, Hubei 430022, P.R. China
\end{abstract}

Received August 9, 2015; Accepted September 26, 2015

DOI: $10.3892 /$ or.2015.4467

\begin{abstract}
Although the tumor suppressive role of miR-101 is well documented in hepatocellular carcinoma (HCC), how the expression of miR-101 itself is regulated remains elusive. In the present study, we demonstrated that the miR-101 precursor pre-miR-101-1 could be regulated by an important epigenetic regulator, the enhancer of zeste homolog 2 (EZH2). Reporter gene assays revealed that ectopic expression of EZH2 inhibited the transcriptional activities of miR-101-1 promoter. Subsequent analyses revealed that miR-101-1 directly represses the expression of EZH2, and miR-101-1 and EZH2 form a reciprocal negative feedback loop as indicated by the fact that ectopic mature miR-101 could induce endogenous pre-miR101-1 expression. This mature miR-101-induced pre-miR-101 expression was specific to pre-miR-101-1 and depended on EZH2 activities. Moreover, our results also demonstrated that similar antitumor effects can be achieved either by ectopic miR-101 or EZH2 silencing in HCC cells. These findings show that elevated EZH2 contributes to miR-101 deregulation in $\mathrm{HCC}$ and highlight the coordinated role of miR-101 and EZH2 in hepatocarcinogenesis.
\end{abstract}

\section{Introduction}

Human hepatocellular carcinoma (HCC) is one of the most common malignant tumors worldwide, particularly prevalent in Africa and Asia. In spite of recent advances in surgical techniques and medical management, the long-term prognosis of patients with HCC remains unsatisfactory. For diagnosis and adequate treatment of $\mathrm{HCC}$, understanding of molecules

Correspondence to: Professor Lihua Hu, Department of Clinical Laboratory, Wuhan Union Hospital, Tongji Medical College, Huazhong University of Science and Technology, 1277 Jiefang Avenue, Wuhan, Hubei 430022, P.R. China

E-mail: huangunion@163.com

*Contributed equally

Key words: enhancer of zeste homolog 2, miR-101, feedback loop, human hepatocellular carcinoma that are responsible for hepatocarcinogenesis and progression is critical (1).

MicroRNAs (miRNAs) are short ( 22 nucleotide), single-stranded, genome-encoded RNA molecules, which are generated by the cleavage of precursor hairpins in two sequential processing reactions. Initially, miRNAs are transcribed as long primary-miRNA (pri-miRNA) transcripts which are cleaved in the nucleus by the enzyme DROSHA (Drosha) to liberate the precursor-miRNA (pre-miRNA) hairpin. The pre-miRNA is subsequently exported from the nucleus and further processed by the enzyme DICER 1 (Dicer) in the cytoplasm to produce mature miRNAs $(2,3)$. These tiny molecules have diverse biological functions. In cancer, the loss of tumor-suppressive miRNAs enhances the expression of target oncogenes, whereas increased expression of oncogenic miRNAs (known as oncomirs) can repress target tumor suppressor genes $(4,5)$.

Recent studies have shown that miRNA expression can be affected by chromosomal abnormalities, mutations, polymorphisms (SNPs), transcriptional deregulation, defects in the miRNA biogenesis machinery and epigenetic changes (6). Enhancer of zeste homolog 2 methyltransferase (EZH2), which is a catalytic subunit of the polycomb repressive complex 2 (PRC2), is responsible for trimethylation of histone $\mathrm{H} 3$ on lysine 27 (H3K27me3), and directly controls DNA methylation (7). It has been shown that EZH2 can be regulated by miR-101 in a plethora of cancers (8-12). Recently, however, several chip-based studies have shown that EZH2 not only regulates protein-encoding genes but also miRNAs $(13,14)$.

miR-101 is encoded by two separate genes in human (miR-101-1 and miR-101-2). $m i R-101-1$ is located in intergenic region and $m i R-101-2$ in the eighth intron of $R C L 1$ gene (15). miR-101 is frequently downregulated in human HCC tissues, and ectopic overexpression of miR-101 markedly induces apoptosis and inhibits proliferation, migration, EMT and angiogenesis in $\mathrm{HCC}$ by targeting multiple target genes such as EZH2, COX-2, STMN1, ROCK2, MCL-1 and FOS (16). Although the antitumor role of miR-101 is well-documented, the transcriptional regulation and the regulatory network of miR-101 remain obscure. In the present study, we found that miR-101 was regulated by EZH2 and they formed a reciprocal negative feedback loop that kept miR-101 in depleted state in HCC. 


\section{Materials and methods}

Cell line and cell culture. A normal hepatic cell line Lo2, and two HCC cell lines, HepG2 and SMMC-7721 were maintained in Dulbecco's modified Eagle's medium (DMEM) supplemented with $10 \%$ fetal bovine serum at $37^{\circ} \mathrm{C}$ with $5 \% \mathrm{CO}_{2}$.

$R T$ - $q P C R$. Pre-miRNA and mature miRNA was extracted with RNAiso for Small RNA (Takara, Dalian, China). The concentration and purity of RNA were controlled using NanoDrop 2000C (Thermo Scientific, Waltham, MA, USA). Mature miR-101 was quantified using the Hairpin-it ${ }^{\mathrm{TM}}$ miRNAs qPCR Quantitation kit (GenePharma, Shanghai, China) according to the manufacturer's instructions. miScript Precursor Assays (Qiagen, Hilden, Germany) were used for pre-miRNA quantification according to the manufacturer's instructions. U6 was used for normalization. EZH2 transcript levels were quantified as described by Guo et al (17). PrimeScript ${ }^{\mathrm{TM}}$ RT reagent kit (Takara) was used for reverse transcription reaction according to the manufacturer's instructions. SYBR-Green RT-qPCR was carried out on StepOnePlus system (Applied Biosystems, Foster City, CA, USA). All reactions were run in triplicate. The relative quantification in gene expression was determined using the $2^{-\Delta \Delta \mathrm{Cq}}$ method.

Construction of plasmids. A 292-bp fragment of EZH2 untranslated region (3'UTR) was amplified by PCR with the following primers: (forward) 5'-ATAGAATTCCATCTGCT ACCTCCTCC-3' and (reverse) 5'-CGCAAGCTTGATTCA ACAAGGAC-3'; and cloned downstream of the firefly luciferase gene in pCMV-Tag2A vector (EZH2-3'UTR) (18). The predicted miR-101 binding sites (Fig. 3C) were mutated by base pair changes using $D p n$ I-mediated site-directed mutagenesis with the following primers: (M1S), CTTCAGGAACC TCGACTGCAGTGGGCAATTTAGAAAA and (M1A), TTTCTAAATTGCCCACTGCAGTCGAGGTTCCTGAA GC; (M2S), TTCTGAATTTGCAAAAGATCTTAAGAAT AATTTATAG and (M2A), TATAAATTATTCTTAAGATCT TTTGCAAATTCAGAAT. To explore the transcriptional regulation of miR-101, a 2118-bp fragment of the miR-101-1 gene promoter (-1904 to 213 relative to the transcription start site of pri-miR-101-1) was amplified by PCR using the following primers: (forward), 5'-GTCGGTACCCACAAAA CCAATCCCCATTGAAGACCACA-3' and (reverse), 5'-ACC AAGCTTGACCAGCACAAATTACAGCAAAGCACCCC-3' The amplified fragment was inserted into the pGL3-basic vector (Promega, Madison, WI, USA) between the KpnI and HindIII sites, named pGL3-101. For EZH2 overexpression, the coding sequence of EZH2 was cloned into the pcDNA3.1, named pcDNA-EZH2, using the following primers: (forward), 5'-ACGGGTACCATCATGGGCCAGACTG-3' and (reverse), 5'-CCGCTCGAGTCAAGGGATTTCCATTTC-3'.Lentivirusmediated mature miR-101 vectors (lenti-miR-101) and control miRNA were constructed and validated by GenePharma.

Cell transfection and luciferase assays. For lenti-miR-101 transfection, HepG2 and SMMC-7721 cells were seeded into 6 -well plates at $3.5 \times 10^{5}$ cells/well. After propagation for $24 \mathrm{~h}$, virus particles $\left(3 \times 10^{7}\right)$ were added. For the reporter assays, HepG2 and SMMC-7721 cells were transfected by
Lipofectamine 2000 (Invitrogen) with $1 \mu \mathrm{g}$ of each constructed vector. For reporter gene assays, $200 \mathrm{ng}$ of the expression or control vectors (pcDNA3.1) was co-transfected with $800 \mathrm{ng}$ of the constructed reporter vector. In each transfection, $50 \mathrm{ng}$ of pRL-TK (Promega) was used to correct for the transfection efficiency. Luciferase activity was measured with the Dual-Luciferase Reporter Assay system (Promega). Promoter activities were expressed as the ratio of Firefly to Renilla luciferase activity. Chemically synthesized RNAs, including negative control (NC), EZH2-siRNA were obtained from GenePharma. For transfection, the cells were transfected with $1 \mu \mathrm{g}$ of the chemically synthesized RNA.

Quantitative genomic PCR for miR-101. DNA was extracted using Takara MiniBEST Universal Genomic DNA Extraction kit (Takara). miScript Precursor Assays (Qiagen) were used for DNA quantification. GAPDH was used for normalization.

5-Aza-2'-deoxycytidine (5-aza-dC) treatment. Cells were treated with 5-aza-2'-deoxycytidine $(1 \mu \mathrm{M})$ (Sigma) for $48 \mathrm{~h}$.

Culture plate colony formation assay. Cells (200) were plated in a fresh 6-well plate and were maintained in complete medium for 15 days. Colonies were fixed with methanol and stained with $0.1 \%$ crystal violet in $20 \%$ methanol for $15 \mathrm{~min}$. Data were obtained from three independent experiments.

Migration and invasion assays. For invasion assays, $1.0 \times 10^{5}$ cells were seeded in a Matrigel-coated chamber with $8.0-\mu \mathrm{m}$ pores (BD Biosciences); for motility assays, $5.0 \times 10^{4}$ cells were plated on top of uncoated membranes with $8.0-\mu \mathrm{m}$ pores. Cells were seeded in serum-free media and translocated toward complete growth media for $24 \mathrm{~h}$. All the experiments were repeated at least three times.

Wound healing assay. Cells grown in a 6-well plate with $90 \%$ confluence were starved in low serum medium $(0.5-0.1 \%$ serum) overnight. A line was drawn with a sterile $200 \mu 1$ pipette tip on the bottom of the well; the cells were rinsed with phosphate-buffered saline (PBS) and cultured in the same medium for $48 \mathrm{~h}$ before photography. Percent migration was calculated by measuring the length and width of the cell-free area. The width was measured at five points along the scratch area and then averaged to get an accurate representation of the entire scratch. Percent migration was determined using the following formula: $[\Delta$ area/area (day 0)] x 100.

Western blot analysis. Total protein was extracted using RIPA buffer (Beyotime, Haimen, China). Equal amounts of protein was separated by $10 \%$ SDS-PAGE and blotted to polyvinylidene fluoride (PVDF) membranes (Millipore, Billerica, MA, USA). Membranes were blocked with 5\% skimmed milk at room temperature for $2 \mathrm{~h}$ and incubated overnight with primary antibodies: anti-EZH2 antibody (1:1,000 dilution; Cell Signaling Technology, Beverly, MA, USA) or anti-GAPDH antibody (1:5,000 dilution; Santa Cruz Biotechnology, Santa Cruz, CA, USA). After three 5 min washes, membranes were incubated with horseradish peroxidase-conjugated secondary antibody (1:5,000 dilution; Cell Signaling Technology). 

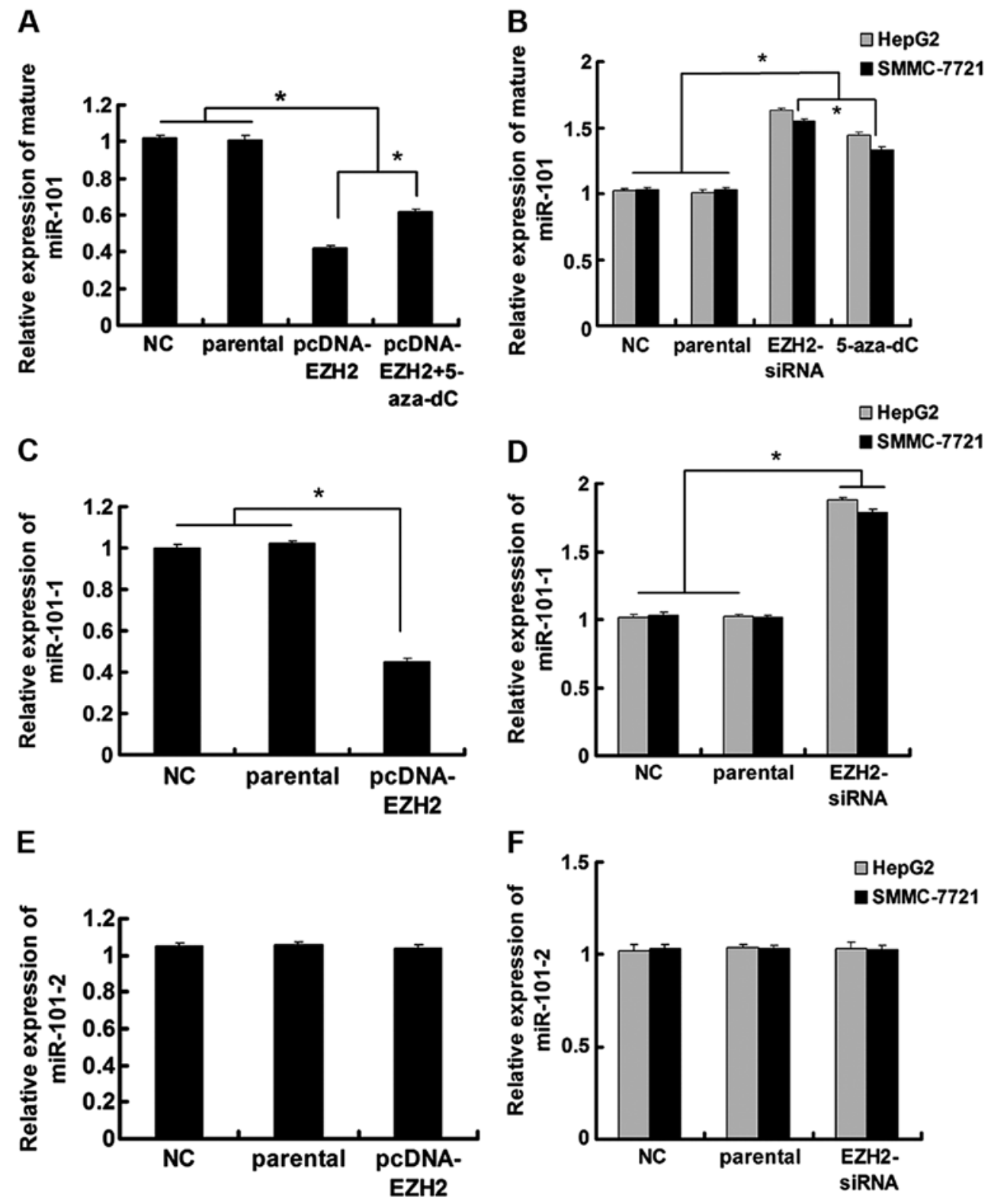

Figure 1. EZH2 negatively regulates miR-1-1. (A) EZH2 overexpression (pcDNA-EZH2) reduced mature miR-101 transcripts in Lo2 cells. Treatment with 5-aza-dC counteracted this EZH2-mediated miR-101 downregulation in Lo2 cells. (B) Treatment with EZH2-siRNA and 5-aza-dC-induced miR-101 expression in HepG2 and SMMC-7721 cell lines. (C) EZH2 overexpression reduced miR-101-1 transcripts in Lo2 cells. (D) Treatment with EZH2-siRNA induced miR-101-1 expression in HepG2 and SMMC-7721 cell lines. (E and F) miR-101-2 was resistant to EZH2 modulation in Lo2 cells (E) or in HepG2 and SMMC-7721 cell lines (F). U6 was used as the reference control for all experiments and the data are from at least three independent experiments, each in triplicate. ${ }^{*} \mathrm{P}<0.05$.

Statistical analysis. Data are presented as mean \pm SD from at least three separate experiments. Unless otherwise noted, one-way ANOVA was used for comparisons between groups. All statistical analyses were carried out with SPSS 12.0 computer software (SPSS, Inc., Chicago, IL, USA). P-values $<0.05$ were considered to indicate a statistically significant result.

\section{Results}

EZH2 negatively regulates miR-101-1 in HCC cell lines. To determine whether EZH2 regulates miR-101 in HCC, we transfected Lo2 cells with EZH2 expression vector (pcDNA-EZH2). RT-qPCR demonstrated a decrease in
miR-101 levels (Fig. 1A). Treatment with the DNA methylation inhibitor 5-aza-2'-deoxycytidine (5-aza-dC) partially inhibited this EZH2-mediated miR-101 downregulation in Lo2 cells (Fig. 1A). In HepG2 and SMMC-7721 cells, treatment with either 5-aza-dC or EZH2-siRNA increased mature miR-101 levels (Fig. 1B).

Since miR-101 has two genomic loci (miR-101-1, on chromosome 1; miR-101-2, on chromosome 9), to pinpoint the locus that is regulated by EZH2, we proceeded to detect miR-101 precursor levels after EZH2 modulation. Our results showed that even though ectopic EZH2 could reduce miR-101-1 expression in Lo2 cells and EZH2 knockdown increased miR-101-1 levels in HepG2 and SMMC-7721 cells, miR-101-2 was resistant to EZH2 modulation (Fig. 1C-F). 
A

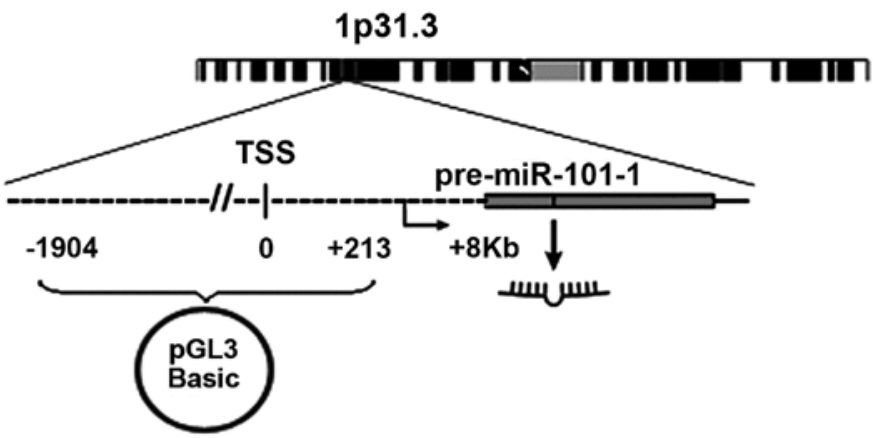

B

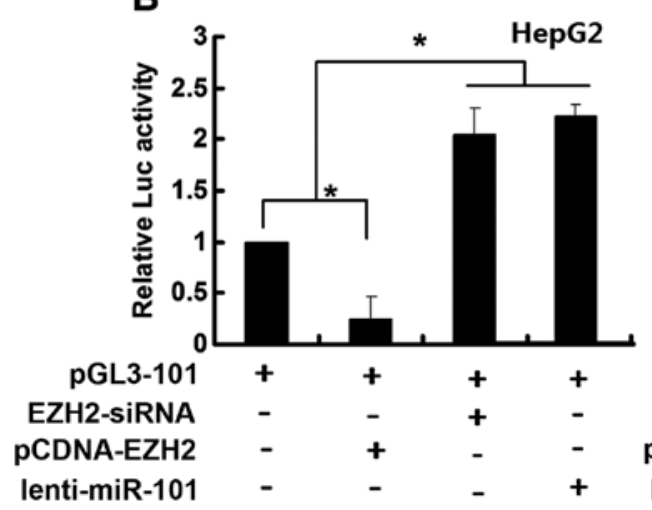

C

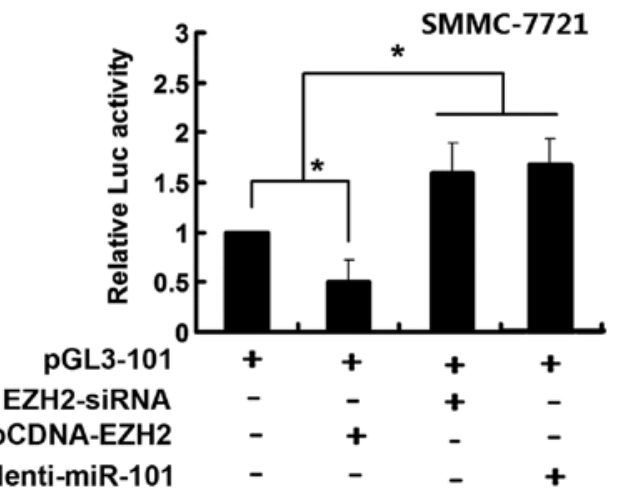

Figure 2. EZH2 knockdown and ectopic miR-101 enhance the transcriptional activity of $m i R-101-1$ promoter. (A) Schematic representation of the upstream region of pre-miR-101-1 and the inserted fragment of pre-miR-101-1 promoter region in pGL3 basic vector. The upstream sequence information of pre-miR-101-1 TSS was acquired from http://mirstart.mbc.nctu.edu.tw. (B and C) Luciferase reporter assays indicated that $m i R-101-1$ promoter region was involved in EZH2dependent miR-101-1 regulation in HepG2 (B) and SMMC-7721 (C) cell lines. Cells were co-transfected with Renilla luciferase expression construct pRL-TK and the indicated DNA constructs. The luciferase activity for the cells that were co-transfected with mock DNA (empty pCDNA3.1) and pGL3-101 vector was set as relative luciferase activity 1 (the first column). Data are from at least three independent experiments, each in triplicate. ${ }^{*} \mathrm{P}<0.05$.

To further corroborate these findings, fragments from position-1904 to position-213 in the miR-101-1 promoter (relative to TSS) were cloned into a luciferase reporter plasmid (pGL3-101) and their transcriptional activity was assessed in HepG2 and SMMC-7721 cells (Fig. 2A). We found that luciferase activity was enhanced by EZH2-siRNA while diminished luciferase activity was observed in the cells transfected with pCDNA-EZH2 (Fig. 2B and C), suggesting that miR-101-1 promoter region was involved in EZH2-dependent miR-101-1 regulation.

miR-101 and EZH2 form a reciprocal negative feedback loop in HCC. EZH2 is a quintessential target of miR-101. If EZH2 is the direct target of miR-101 in HCC, it seems to follow that miR-101 and EZH2 may form a reciprocal negative feedback loop in HCC. To test the hypothesis, we transfected HepG2 and SMMC-7721 cells with lentivirus-mediated mature miR-101 vectors (lenti-miR-101) and a control miRNA. Our results showed ectopic miR-101 decreased EZH2 expression at both mRNA and protein level (Fig. 3A and B). To test whether the 3'UTR of EZH2 was responsible for its regulation, we cloned EZH2 3'UTR downstream of pCMV-Tag2A-luciferase reporter gene and constructed corresponding mutated reporter vectors (Fig. 3C). Renilla luciferase vector (pRL-TK) was used as reference control. Reporter gene assays showed that the luciferase activity was significantly decreased by ectopic miR-101 as compared with negative control while the mutation in the predicted target sites abolished the repressive effects of miR-101 on luciferase activity (Fig. 3D), suggesting that miR-101 regulated EZH2 expression by binding to its target sites at the 3'UTR of EZH2.

To prove the existence of EZH2/miR-101-1 feedback loop, we tested the inducibility of precursor miR-101s by mature miR-101. Compared with parental cell lines, the pre-miR-101-1 levels of HepG2 and SMMC-7721 cells witnessed a 2- to 4-fold increase after ectopic expression of mature miR-101, while co-transfection of lenti-miR-101 with pcDNA-EZH2 could reduce the pre-miR-101-1-inducing effects of ectopic mature miR-101 (Fig. 4A). To further corroborate this gain-of-function study, we co-transfected pGL3-101 with lenti-miR-101 in HepG2 and SMMC-7721 cells. Our results indicated that ectopic miR-101 could enhance the transcriptional activity of $m i R-101-1$ promoter (Fig. 2B and C).

To address the issue of why HepG2 and SMMC-7721 cells had different induction efficiencies, we performed quantitative genomic PCR for miR-101-1, as the inducibility of pre-miRNA by ectopic mature miRNA depends on the integrity of miRNA loci. Compared with Lo2 cells, Both HepG2 and SMMC-7721 cells exhibited miR-101-1 locus deletion. However, SMMC-7721 cells showed much heavier miR-101-1 locus deletion than HepG2 cells (Fig. 4B). These results suggest that the lower inducibility of pre-miR-101-1 in SMMC-7721 cells may be correlated with heavier miR-101-1 locus deletion. Taken together, our results suggest that EZH2 
A

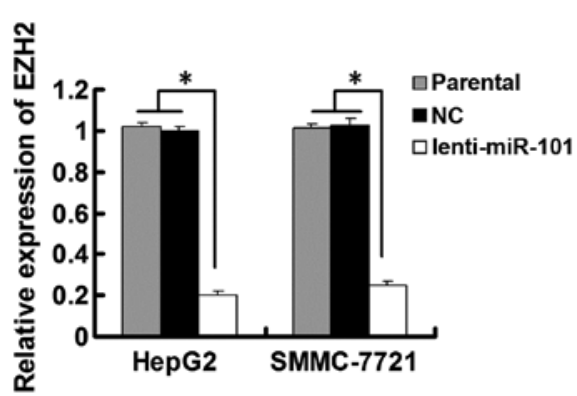

C

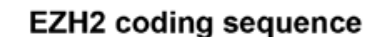

B

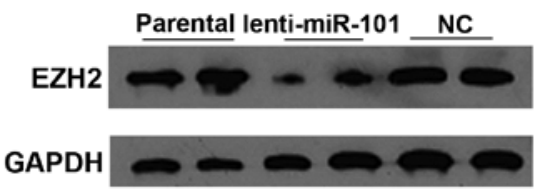

EZH2-3'UTR

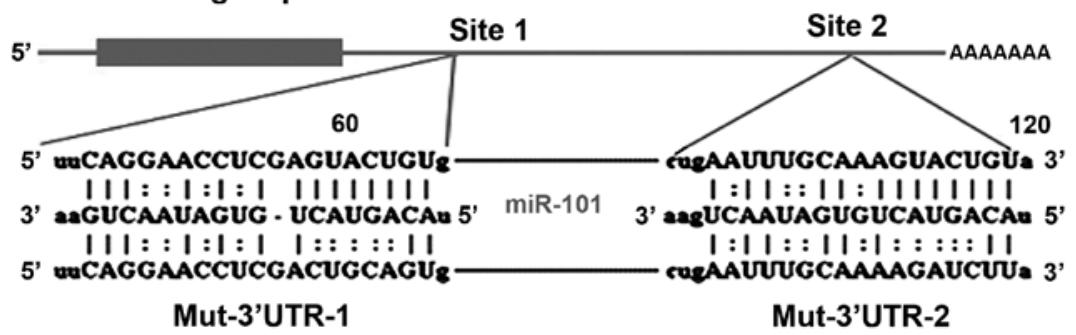

D
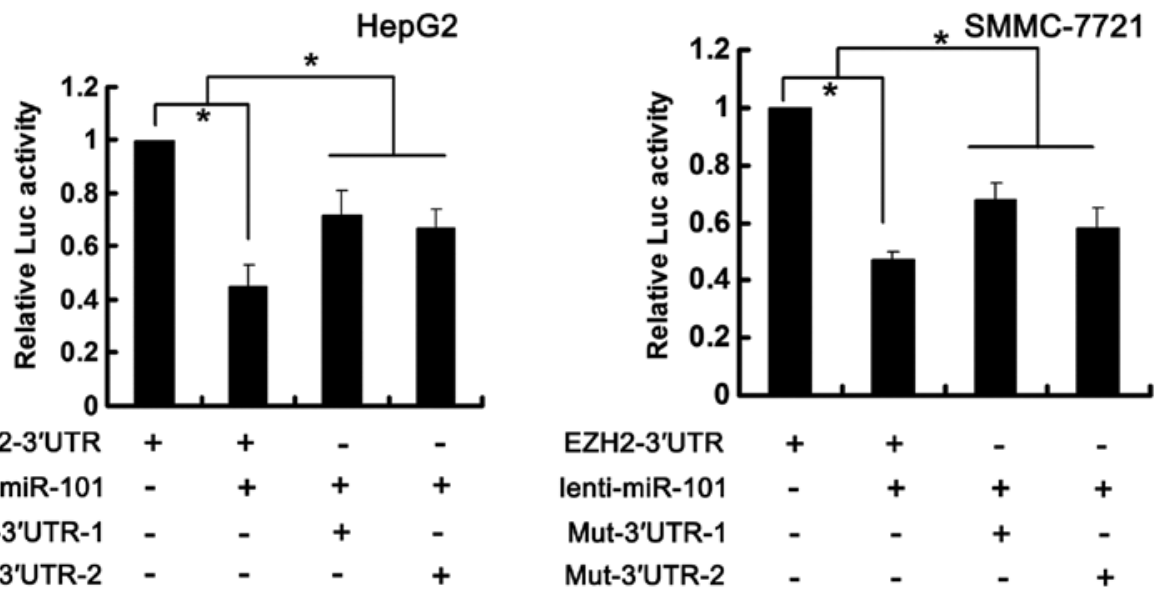

Figure 3. miR-101 negatively regulates EZH2 in HCC cell lines. (A) EZH2 mRNA was reduced by ectopic miR-101 (lenti-miR-101) in HepG2 and SMMC-7721 cell lines. GAPDH was used as the reference control. (B) Ectopic miR-101 (lenti-miR-101) reduced EZH2 expression at protein levels in HepG2 and SMMC-7721 cell lines. (C) Schematic representation of the predicted binding sites for miR-101 in the 3'UTR of EZH2 and the site mutagenesis design for the reporter gene assays. Complementarities between miR-101 and the target sites are shown. (D) Luciferase reporter assays validated EZH2 as a direct target of miR-101. Cells were co-transfected with Renilla luciferase expression construct pRL-TK and indicated DNA constructs. The luciferase activity for the cells that were co-transfected with mock DNA and EZH2-3'UTR was set as relative luciferase activity 1 (the first column). Data are from at least three independent experiments, each in triplicate. ${ }^{*} \mathrm{P}<0.05$.
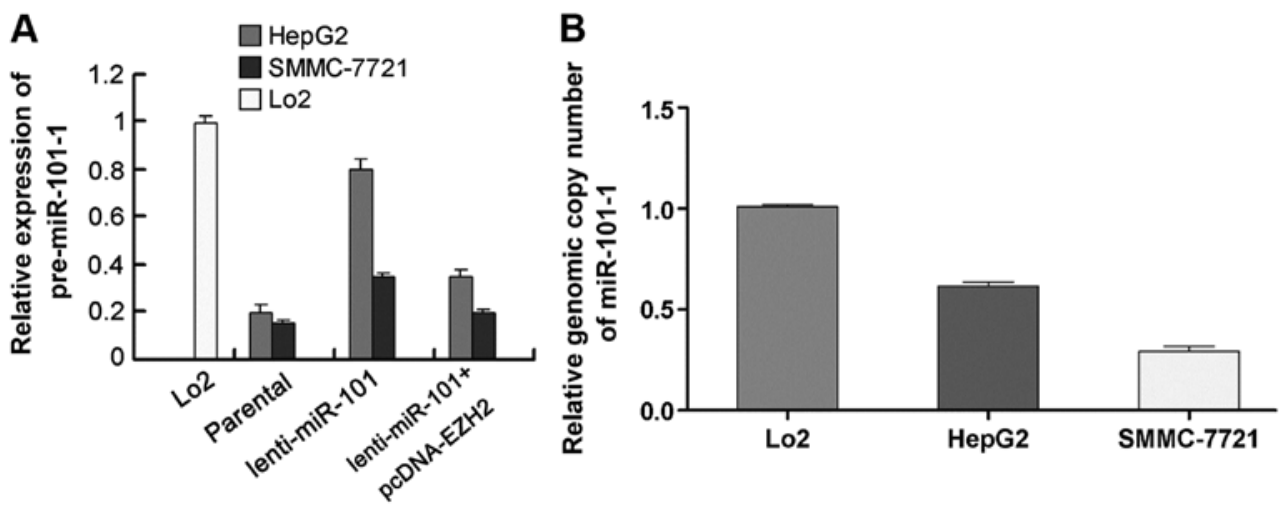

Figure 4. EZH2-dependent induction of endogenous pre-miR101-1 by ectopic mature miR-101. (A) Ectopic mature miR-101-induced pre-miR-101-1 expression in HepG2 and SMMC-7721 cells. For HepG2 cells, the induction restored pre-miR-101-1 levels almost to those observed in immortalized hepatocytes, Lo2 cells. Co-expression of EZH2 counteracted the pre-miR-101-1-inducing effects of ectopic mature miR-101 in HepG2 and SMMC-7721 cells. (B) Quantitative genomic PCR revealed that SMMC-7721 cells had more frequent losses of miR-101-1 loci than the HepG2 cells. Data are from at least three independent experiments, each in triplicate. 
A
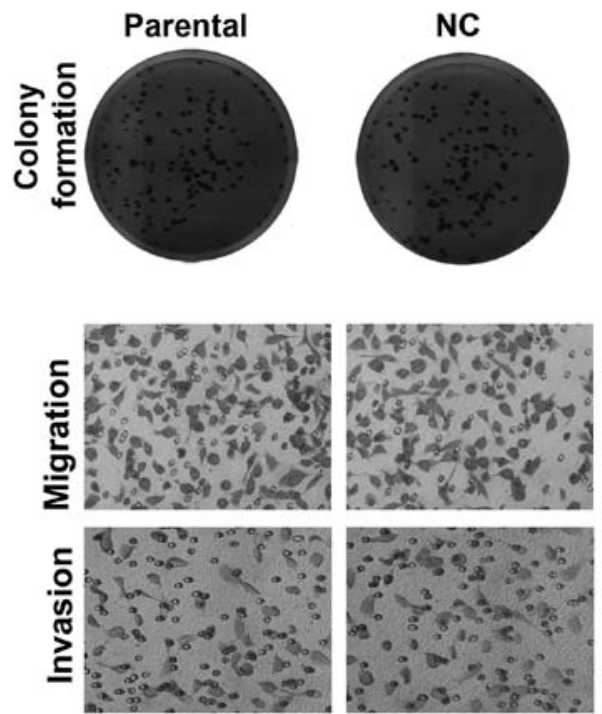
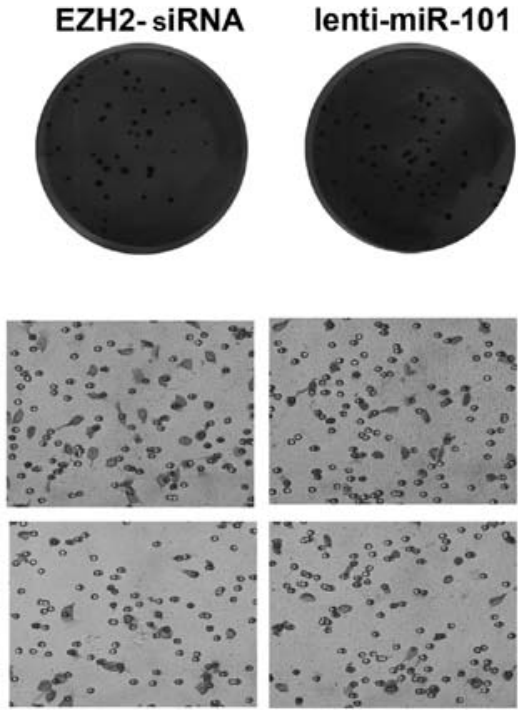

\section{Parental} NC
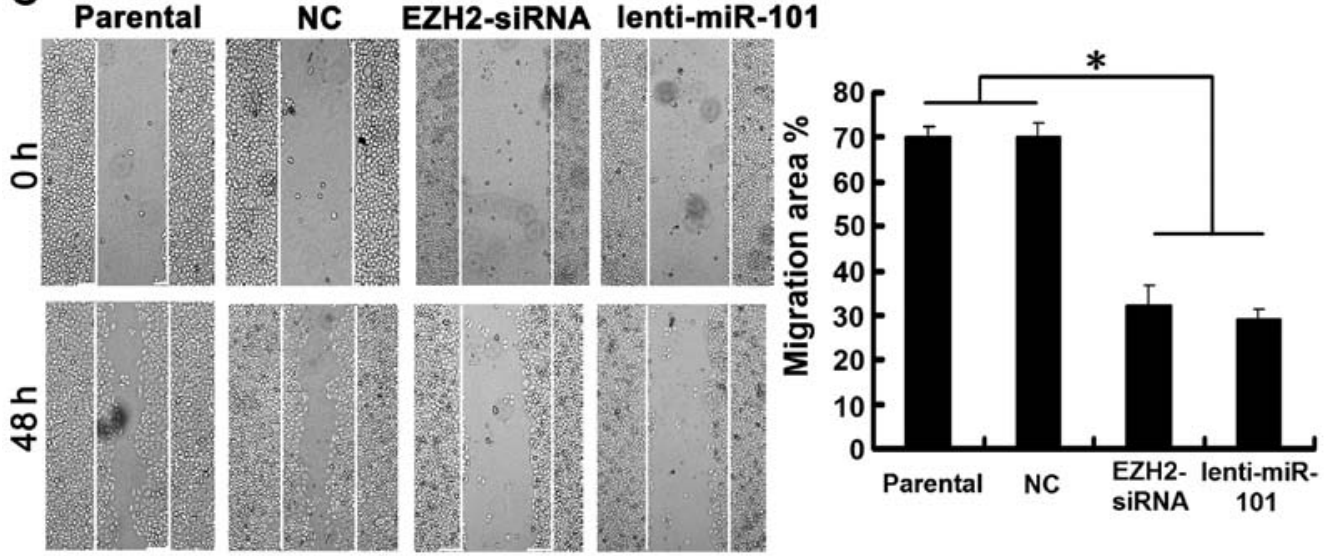

Figure 5. Targeting EZH2/miR-101 loop impedes HCC. (A) Restoration of miR-101 or EZH2 knockdown suppressed colony formation of HepG2 cells. (B) Decreased migration and invasion were observed in HepG2 cells on miR-101 re-expression or EZH2 knockdown. (C) Wound healing assays revealed decelerated wound closure rates in HepG2 cells transfected with EZH2-siRNA or lenti-miR-101. Data are from at least three independent experiments. "P<0.05.

and miR-101 negatively regulates each other and form a feedback loop in HCC.

Manipulation of EZH2/miR-101 feedback loop exerts antitumor effects. To demonstrate the efficacy of targeting EZH2/miR-101-1 feedback loop in impeding HCC, we transfected HepG2 cells with lenti-miR-101 or EZH2-siRNA. Our results showed that cells displayed much fewer and smaller colonies compared with NC transfectants and parental cells (Fig. 5A). Moreover, Transwell migration and invasion assays revealed that cells transfected with lenti-miR-101 or EZH2-siRNA demonstrated a decreased ability to pass through $8-\mu \mathrm{m}$ pore size polycarbonate membrane compared with NC transfectants and parental cells (Fig. 5B). The effects of ectopic miR-101 and EZH2 silencing on cell migration was further supported by wound healing assays (Fig. 5C). Taken together, our results suggest that similar antitumor effects can be achieved both by ectopic miR-101 or EZH2 silencing in HCC cells and manipulation of this miRNA feedback loop may have the therapeutic potential for treating liver cancer.

\section{Discussion}

miRNA genes constitute one of the most abundant gene families, and are widely distributed in animals, plants and viruses. The latest release of the miRNA database (miRBase) has catalogued 2,588 miRNAs in humans. Targeting most protein-coding transcripts, miRNAs are involved in nearly all developmental and pathological processes in animals. The biogenesis of miRNAs is under tight temporal and spatial control, and their dysregulation is associated with many human diseases, particularly cancer. miRNA regulation takes place at multiple steps, including their transcription, their processing by Drosha and Dicer, their loading onto AGO proteins and miRNA turnover $(19,20)$.

Downregulation of miR-101 is a common event in cancers and has been implicated in the development and progression of different malignancies. Previous studies show genomic loss of miR-101 is one of the main mechanisms of decreased miR-101 expression in prostate, breast and gastric cancers, and glioblastoma (15). Our genomic PCR results indicate that this mechanism also plays a role in HCC, since allele losses 
were observed in HepG2 and SMMC-7721 cells. However, cancer cells are a heterogeneous population and no more than half of the cases of HCC were found to have miR-101 locus losses in previous comparative genome hybridization studies (21-23). How cancer cells that have intact miR-101 loci also exhibit miR-101 depletion is largely unsolved. A previous study shows that activator protein-1 (AP-1) directly binds to the -17.4 to $-16.4 \mathrm{k}$ region upstream of pre-miR-101-2 and activated the expression of miR-101 (15). In the present study, we report that miR-101 expression can be regulated by the key epigenetic regulator EZH2 in $m i R$-101-1-intact HCC cells and $m i R-101-1$ promoter region is involved in this process. Moreover, our results show that EZH2-meditated regulation of miR-101 is not simply a 'one-way relationship'. Instead, they form a reciprocal negative feedback loop: high levels of EZH2 contribute to the depletion of miR-101, which, in turn, helps HCC cells keep EZH2 at high levels, hence sustained miR-101 silencing. Several recent studies have reported similar feedback networks that play critical roles in cancer (24-26). These studies, along with the present one, strongly suggest that feedback networks involving miRNAs and their targets may represent a common mechanism in cancer development.

Theoretically, EZH2 may regulate miR-101-1 at the transcription or processing steps. Our evidence, however, indicates that the regulation takes place at the transcription step rather than the processing step. First, miRNA-101-2 and miRNA-101-1 share a common processing pathway except that miRNA-101-2, being an intragenic miRNA (in the eighth intron of $R C L 1$ gene), does not require Drosha for cleavage once it is co-transcribed with the host gene. In this regard, the resistance of pre-miR-101-2 to induction by EZH2 suggests the regulation of miR-101-1 by EZH2 should take place prior to Dicer cleavage. Second, our reporter gene assays showed that ectopic expression of EZH2 inhibited the transcriptional activities of $m i R-101-1$ promoter. $m i R-101-1$ promoter region has multiple $\mathrm{CpG}$ islands with high GC content (>90\%). This GC-rich region had defied all our attempts to amplify it using routine PCR methods such as adding organic additives and jointly using highly effective DNA polymerase or even slowdown PCR $(27,28)$. We finally managed to amplify this DNA fragment using long primers with high $\mathrm{T}(\mathrm{m})$ and low $\Delta \mathrm{T}(\mathrm{m})(29)$.

miRNA transcription is carried out by RNA Pol II and is controlled by RNA Pol II-associated transcription factors and epigenetic regulators. Transcription factors, such as p53, MYC, ZEB1 and ZEB2, and myoblast determination protein 1 (MYOD1) positively or negatively regulate miRNA expression (30). Epigenetic control, such as DNA methylation and histone modifications also contribute to miRNA gene regulation (31). In this regard, $\mathrm{EZH} 2$, which is responsible for trimethylation of histone $\mathrm{H} 3$ on lysine 27 (H3K27me3) and directly controls DNA methylation, may regulate miR-101-1 expression in two possible ways. That is, it can directly regulate $m i R-101-1$ promoter by DNA methylation and histone modification or indirectly via regulating transcription factor(s). However, which mode of regulation is the case in HCC should be further studied.

Another property of reciprocal negative feedback loop is that it allows the system to remain reversible. This property means targeting the feedback circuit at any level (miR-101 overexpression or EZH2 silencing) would trigger auto-amplification reactions and bring about stable antitumor effects. Consistent with this concept, we found that ectopic miR-101 and EZH2-siRNA had similar antitumor effects on HCC in vitro. In summary, we have deciphered a feedback mechanism that controls miR-101 expression. The finding of this mechanism sheds new light on hepatocarcinogenesis. Our data also raise the possibility that manipulation of this microRNA feedback loop has the therapeutic potential for treating liver cancer.

\section{Acknowledgements}

The present study was supported by grants 81001065 from the National Natural Science Foundation of China.

\section{References}

1. El-Serag HB and Rudolph KL: Hepatocellular carcinoma: Epidemiology and molecular carcinogenesis. Gastroenterology 132: 2557-2576, 2007.

2. Lee Y, Ahn C, Han J, Choi H, Kim J, Yim J, Lee J, Provost P, Rådmark O, Kim S, et al: The nuclear RNase III Drosha initiates microRNA processing. Nature 425: 415-419, 2003.

3. Lund E, Güttinger S, Calado A, Dahlberg JE and Kutay U: Nuclear export of microRNA precursors. Science 303: 95-98, 2004.

4. Bracken CP, Khew-Goodall Y and Goodall GJ: Network-Based Approaches to Understand the Roles of miR-200 and Other microRNAs in Cancer. Cancer Res 75: 2594-2599, 2015.

5. Lujambio A and Lowe SW: The microcosmos of cancer. Nature 482: 347-355, 2012.

6. Iorio $\mathrm{MV}$, Piovan $\mathrm{C}$ and Croce $\mathrm{CM}$ : Interplay between microRNAs and the epigenetic machinery: An intricate network. Biochim Biophys Acta 1799: 694-701, 2010.

7. Viré E, Brenner C, Deplus R, Blanchon L, Fraga M, Didelot C, Morey L, Van Eynde A, Bernard D, Vanderwinden JM, et al: The Polycomb group protein EZH2 directly controls DNA methylation. Nature 439: 871-874, 2006.

8. Sakurai T, Bilim VN, Ugolkov AV, Yuuki K, Tsukigi M, Motoyama T and Tomita Y: The enhancer of zeste homolog 2 $(\mathrm{EZH} 2)$, a potential therapeutic target, is regulated by miR-101 in renal cancer cells. Biochem Biophys Res Commun 422: 607-614, 2012.

9. Alajez NM, Shi W, Hui AB, Bruce J, Lenarduzzi M, Ito E, Yue S, O'Sullivan B and Liu FF: Enhancer of Zeste homolog 2 (EZH2) is overexpressed in recurrent nasopharyngeal carcinoma and is regulated by miR-26a, miR-101, and miR-98. Cell Death Dis 1: e85, 2010.

10. Zhang JG, Guo JF, Liu DL, Liu Q and Wang JJ: MicroRNA-101 exerts tumor-suppressive functions in non-small cell lung cancer through directly targeting enhancer of zeste homolog 2. J Thorac Oncol 6: 671-678, 2011.

11. Cho HM, Jeon HS, Lee SY, Jeong KJ, Park SY, Lee HY, Lee JU, Kim JH, Kwon SJ, Choi E, et al: microRNA-101 inhibits lung cancer invasion through the regulation of enhancer of zeste homolog 2. Exp Ther Med 2: 963-967, 2011.

12. Ren G, Baritaki S, Marathe H, Feng J, Park S, Beach S, Bazeley PS, Beshir AB, Fenteany G, Mehra R, et al: Polycomb protein $\mathrm{EZH} 2$ regulates tumor invasion via the transcriptional repression of the metastasis suppressor RKIP in breast and prostate cancer. Cancer Res 72: 3091-3104, 2012.

13. Au SL, Wong CC, Lee JM, Fan DN, Tsang FH, Ng IO and Wong CM: Enhancer of zeste homolog 2 epigenetically silences multiple tumor suppressor microRNAs to promote liver cancer metastasis. Hepatology 56: 622-631, 2012.

14. Cao Q, Mani RS, Ateeq B, Dhanasekaran SM, Asangani IA, Prensner JR, Kim JH, Brenner JC, Jing X, Cao X, et al: Coordinated regulation of polycomb group complexes through microRNAs in cancer. Cancer Cell 20: 187-199, 2011.

15. Liu JJ, Lin XJ, Yang XJ, Zhou L, He S, Zhuang SM and Yang J: A novel AP-1/miR-101 regulatory feedback loop and its implication in the migration and invasion of hepatoma cells. Nucleic Acids Res 42: 12041-12051, 2014. 
16. Zheng F, Liao YJ, Cai MY, Liu TH, Chen SP, Wu PH, Wu L, Bian XW, Guan XY, Zeng YX, et al: Systemic delivery of microRNA-101 potently inhibits hepatocellular carcinoma in vivo by repressing multiple targets. PLoS Genet 11: e1004873, 2015.

17. Guo J, Cai J, Yu L, Tang H, Chen C and Wang Z: EZH2 regulates expression of $\mathrm{p} 57$ and contributes to progression of ovarian cancer in vitro and in vivo. Cancer Sci 102: 530-539, 2011.

18. Li Y, Xie J, Xu X, Wang J, Ao F, Wan Y and Zhu Y: MicroRNA-548 down-regulates host antiviral response via direct targeting of IFN- $\lambda 1$. Protein Cell 4: 130-141, 2013.

19. Ha M and Kim VN: Regulation of microRNA biogenesis. Nat Rev Mol Cell Biol 15: 509-524, 2014.

20. Krol J, Loedige I and Filipowicz W: The widespread regulation of microRNA biogenesis, function and decay. Nat Rev Genet 11: 597-610, 2010.

21. Qin LX, Tang ZY, Sham JS, Ma ZC, Ye SL, Zhou XD, Wu ZQ, Trent JM and Guan XY: The association of chromosome $8 \mathrm{p}$ deletion and tumor metastasis in human hepatocellular carcinoma. Cancer Res 59: 5662-5665, 1999.

22. Leung TH, Wong N, Lai PB, Chan A, To KF, Liew CT, Lau WY and Johnson PJ: Identification of four distinct regions of allelic imbalances on chromosome 1 by the combined comparative genomic hybridization and microsatellite analysis on hepatocellular carcinoma. Mod Pathol 15: 1213-1220, 2002.

23. Patil MA, Gütgemann I, Zhang J, Ho C, Cheung ST, Ginzinger D, Li R, Dykema KJ, So S, Fan ST, et al: Array-based comparative genomic hybridization reveals recurrent chromosomal aberrations and Jab1 as a potential target for 8q gain in hepatocellular carcinoma. Carcinogenesis 26: 2050-2057, 2005.
24. Brabletz T: MiR-34 and SNAIL: Another double-negative feedback loop controlling cellular plasticity/EMT governed by p53. Cell Cycle 11: 215-216, 2012.

25. Luzi E, Marini F, Giusti F, Galli G, Cavalli L and Brandi ML: The negative feedback-loop between the oncomir Mir-24-1 and menin modulates the Men1 tumorigenesis by mimicking the 'Knudson's second hit'. PLoS One 7: e39767, 2012.

26. Yamakuchi M and Lowenstein CJ: MiR-34, SIRT1 and p53: The feedback loop. Cell Cycle 8: 712-715, 2009.

27. Bachmann HS, Siffert W and Frey UH: Successful amplification of extremely GC-rich promoter regions using a novel 'slowdown PCR' technique. Pharmacogenetics 13: 759-766, 2003.

28. Frey UH,Bachmann HS, Peters J and Siffert W: PCR-amplification of GC-rich regions: 'Slowdown PCR'. Nat Protoc 3: 1312-1317, 2008.

29. Li LY, Li Q, Yu YH, Zhong M, Yang L, Wu QH, Qiu YR and Luo SQ: A primer design strategy for PCR amplification of GC-rich DNA sequences. Clin Biochem 44: 692-698, 2011.

30. Kim VN, Han J and Siomi MC: Biogenesis of small RNAs in animals. Nat Rev Mol Cell Biol 10: 126-139, 2009.

31. Davis-Dusenbery BN and Hata A: Mechanisms of control of microRNA biogenesis. J Biochem 148: 381-392, 2010. 